\title{
Cycle Chains and the LPA Model
}

\author{
J.M. CUSHING \\ Department of Mathematics, University of Arizona, 617 N. Santa Rita, Tucson, AZ 85721, USA
}

(Received 19 April 2002; In final form 4 September 2002)

The LPA model is a three dimensional system of nonlinear difference equations that has found many applications in population dynamics and ecology. In this paper, we consider a special case of the model (a case that approximates that used in experimental bifurcation and chaos studies) and prove several theorems concerning the existence and stability of periodic cycles, invariant loops, and chaos. Key is the notion of synchronous orbits (i.e. orbits lying on the coordinate planes). The main result concerns the existence of an invariant loop of synchronous orbits that bifurcates, in a nongeneric way, from the trivial equilibrium. The geometry and dynamics of this invariant loop are characterized. Specifically, it is shown that the loop is a cycle chain consisting of synchronous heteroclinic orbits connecting the three temporal phases of a synchronous 3-cycle. We also show that a period doubling route to chaos occurs within the class of synchronous orbits.

Keywords: Difference equations; Stability; Bifurcation; Equilibria; Periodic cycles; Invariant loop

AMS 2000 Mathematics Subject Classification: 39A11; 92B05

\section{INTRODUCTION}

The system of difference equations

$$
\begin{aligned}
& L_{t+1}=b A_{t} \exp \left(-c_{e l} L_{t}-c_{e a} A_{t}\right) \\
& P_{t+1}=\left(1-\mu_{l}\right) L_{t} \\
& A_{t+1}=P_{t} \exp \left(-c_{p a} A_{t}\right)+\left(1-\mu_{a}\right) A_{t} \\
& L_{0} \geq 0, \quad P_{0} \geq 0, \quad \mathrm{~A}_{0} \geq 0
\end{aligned}
$$

is known as the LPA model. All coefficients are positive and $\mu_{l}<1, \mu_{a} \leq 1$. In applications to population dynamics, these equations describe the time evolution of an orbit of demographic triples

$$
\left(\begin{array}{l}
L_{t} \\
P_{t} \\
A_{t}
\end{array}\right)
$$


consisting of larval, pupal, and adult population densities. As indicated by the initial conditions in Eq. (1), only orbits lying in the nonnegative cone are of interest in applications.

The LPA model has been extraordinarily successful in experimental studies of complex nonlinear phenomena that occur in the dynamics of laboratory insects (specifically, flour beetles of the genus Tribolium ) $[3,8,13,17]$. Although many of these studies deal with the asymptotic dynamics of the LPA model, a great deal remains unknown, from a rigorous mathematical point of view, about the attractors, stability, and bifurcations exhibited by the Eq. (1). The following is a brief summary of the mathematical facts known about the system (1) (at least to the author). See Ref. [6] for more details.

All orbits defined by Eq. (1) are nonnegative and bounded for $t \geq 0$. More technically, the map defined by these equations leaves the nonnegative cone invariant and is dissipative (i.e. within finitely many steps each orbit lies in a specified compact set). If the positive number

$$
n=\frac{b\left(1-\mu_{l}\right)}{\mu_{a}}
$$

(known as the inherent net reproductive number) is less than 1, then the "trivial" equilibrium

$$
\left(\begin{array}{l}
L \\
P \\
A
\end{array}\right)=\left(\begin{array}{l}
0 \\
0 \\
0
\end{array}\right)
$$

is locally asymptotically stable (LAS) and attracts all orbits in the nonnegative cone. As $n$ increases above 1, a "primary" bifurcation occurs which results in the instability of the trivial equilibrium and the creation of a positive equilibrium. In fact, for all $n>1$ there exists one and only one positive equilibrium. Although the positive equilibrium may not be stable, the system (1) is uniformly persistent with respect to the origin. That is to say, there is a constant $\delta>0$ such that each orbit satisfies $\liminf _{t \rightarrow \infty}\left(\left|L_{t}\right|+\left|P_{t}\right|+\left|A_{t}\right|\right) \geq \delta$. Thus, when $n>1$ no orbit tends to the trivial equilibrium and, in the population dynamic context, populations never go extinct. Only in some special cases is it known when the positive equilibrium is stable. If $n>1, \mu_{a}<1$, and $n-1$ is sufficiently close to 0 , then the positive equilibrium is LAS. This is an example of a generic transcritical bifurcation of equilibria in which there is an exchange of stability from one branch (the trivial equilibrium) to the other (the positive equilibria). If $c_{e l}=0$ the exact parameter regions for which the positive equilibrium is LAS are known, as are the types of bifurcations that occur when the boundaries of these regions are crossed [9]. Also in this case it is known that the positive equilibrium is globally attracting if $n<e \min \left\{1,\left(c_{e a} / c_{p a}\right)\left(\left(1-\mu_{a}\right) / \mu_{a}\right)\right\}$ [15]. (The case $c_{e l}=0$ is not, however, pertinent to populations of flour beetles [8-10].)

In this paper, we study a special case of the LPA model that is closely related to many of the applications that have been made to laboratory cultures of flour beetles. In several long term experiments concerned with complex population dynamics (reported, for example, in Refs. $[4,7,8,10])$ the adult death rate was manipulated to equal $96 \%$ and hence $\mu_{a}=0.96$. Motivated by this fact, we consider the LPA model (1) with $\mu_{a}=1$. Biologically, this means no adults survive longer than one unit of time (two weeks in the case of applications to flour beetles). When $\mu_{a}=1$ the inherent net reproductive number is

$$
n=b\left(1-\mu_{l}\right) \text {. }
$$


and the LPA equation (1) become

$$
\begin{aligned}
& L_{t+1}=n \frac{1}{1-\mu_{l}} A_{t} \exp \left(-c_{e l} L_{t}-c_{e a} A_{t}\right) \\
& P_{t+1}=\left(1-\mu_{l}\right) L_{t} \\
& A_{t+1}=P_{t} \exp \left(-c_{p a} A_{t}\right) \\
& L_{0} \geq 0, \quad P_{0} \geq 0, \quad A_{0} \geq 0 .
\end{aligned}
$$

We show in the next section that the primary bifurcation at $n=1$ for this special case is nongeneric and that an exchange of equilibrium stability does not occur. In the following sections, we show this is due to the simultaneous bifurcation of the positive equilibrium and an invariant loop, and we characterize the geometry and dynamics of this loop.

\section{EQUILIBRIA}

As remarked above, it is known that all orbits of Eq. (2) tend to the (LAS) trivial equilibrium if $n<1$.

THEOREM 1 For $n>1$ the trivial equilibrium of Eq. (2) is unstable (it is a repellor), and there exists a unique positive equilibrium. This positive equilibrium, which bifurcates from the trivial equilibrium at $n=1$, is unstable for $n-1>0$ sufficiently small.

Proof As remarked in the "Introduction" Section, the first sentence is known [6] and hence we have only to prove the second sentence. We do this using Liapunov-Schmidt expansions for the positive equilibrium and for the eigenvalues the Jacobian evaluated at the equilibrium. Details of this method and its rigorous justification can be found in Ref. [6]. If we substitute the expansions

$$
\begin{aligned}
& L(\varepsilon)=L(0) \varepsilon+L^{\prime}(0) \varepsilon^{2}+O\left(\varepsilon^{3}\right) \\
& P(\varepsilon)=P(0) \varepsilon+P^{\prime}(0) \varepsilon^{2}+O\left(\varepsilon^{3}\right) \\
& A(\varepsilon)=A(0) \varepsilon+A^{\prime}(0) \varepsilon^{2}+O\left(\varepsilon^{3}\right) \\
& n(\varepsilon)=1+n_{1} \varepsilon+n_{2} \varepsilon^{2}+O\left(\varepsilon^{3}\right)
\end{aligned}
$$

into the equilibrium equations

$$
\begin{aligned}
L & =n \frac{1}{1-\mu_{l}} A \exp \left(-c_{e l} L-c_{e a} A\right) \\
P & =\left(1-\mu_{l}\right) L \\
A & =P \exp \left(-c_{p a} A\right)
\end{aligned}
$$

for Eq. (2) we obtain, by equating the coefficients of the lowest order terms in $\varepsilon$, the linear algebraic equations

$$
\begin{aligned}
L(0) & =\frac{1}{1-\mu_{l}} A(0) \\
P(0) & =\left(1-\mu_{l}\right) L(0) \\
A(0) & =P(0)
\end{aligned}
$$


for the leading coefficients. Solving these equations, we obtain $L(0)=1, P(0)=1-\mu_{l}$, and $A(0)=1-\mu_{l}$ (up to a constant multiple). By equating the coefficients of the $\varepsilon^{2}$ terms we obtain the algebraic equations.

$$
\begin{aligned}
& L^{\prime}(0)=\frac{1}{1-\mu_{l}} A^{\prime}(0)+n_{1}-c_{e l}-c_{e a}\left(1-\mu_{l}\right) \\
& P^{\prime}(0)=\left(1-\mu_{l}\right) L^{\prime}(0) \\
& A^{\prime}(0)=P^{\prime}(0)-c_{p a}\left(1-\mu_{l}\right)^{2}
\end{aligned}
$$

which constitute a nonhomogeneous version of the linear system (3). Because Eq. (3) is singular, Eq. (4) is solvable if and only if the nonhomogeneous terms are orthogonal to the nullspace of the adjoint to Eq. (3). This condition determines $n_{1}$. Specifically, it turns out that Eq. (4) is solvable if and only if

$$
n_{1}=c_{e l}+\left(c_{e a}+c_{p a}\right)\left(1-\mu_{l}\right)
$$

With this choice for $n_{1}$ we could solve for $L^{\prime}(0), P^{\prime}(0)$, and $A^{\prime}(0)$. However, since we do not need these coefficients for the stability analysis we will not trouble to do this calculation. The expansions

$$
\begin{aligned}
& L(\varepsilon)=\varepsilon+O\left(\varepsilon^{2}\right) \\
& P(\varepsilon)=\left(1-\mu_{l}\right) \varepsilon+O\left(\varepsilon^{2}\right) \\
& A(\varepsilon)=\left(1-\mu_{l}\right) \varepsilon+O\left(\varepsilon^{2}\right) \\
& n(\varepsilon)=1+\left[c_{e l}+\left(c_{e a}+c_{p a}\right)\left(1-\mu_{l}\right)\right] \varepsilon+O\left(\varepsilon^{2}\right)
\end{aligned}
$$

are sufficient for us to determine the local stability of the positive equilibrium. When these expansions are substituted into the Jacobian matrix

$$
J=\left(\begin{array}{ccc}
-c_{e l} n \frac{1}{1-\mu_{l}} A e^{-c_{e a} A-c_{e l} L} & 0 & \left(1-c_{e a} A\right) n \frac{1}{1-\mu_{l}} e^{-c_{e a} A-c_{e l} L} \\
1-\mu_{l} & 0 & 0 \\
0 & e^{-c_{p a} A} & -c_{p a} P e^{-c_{p a} A}
\end{array}\right)
$$

of Eq. (2), the resulting matrix has an expansion of the form

$$
J(\varepsilon)=J(0)+J^{\prime}(0) \varepsilon+O\left(\varepsilon^{2}\right)
$$

where

$$
\begin{aligned}
J(0) & =\left(\begin{array}{ccc}
0 & 0 & \frac{1}{1-\mu_{l}} \\
1-\mu_{l} & 0 & 0 \\
0 & 1 & 0
\end{array}\right) \\
J^{\prime}(0) & =\left(\begin{array}{ccc}
-c_{e l} & 0 & c_{p a}-c_{e a} \\
0 & 0 & 0 \\
0 & -c_{p a}\left(1-\mu_{l}\right) & -c_{p a}\left(1-\mu_{l}\right)
\end{array}\right)
\end{aligned}
$$


Each of the three eigenvalues $\sigma_{i}=\sigma_{i}(\varepsilon)$ and associated eigenvectors $v_{i}=v_{i}(\varepsilon)$ of $J(\varepsilon)$ also have $\epsilon$ expansions

$$
\begin{gathered}
\sigma_{i}(\varepsilon)=\sigma_{i}(0)+\sigma_{i}^{\prime}(0) \varepsilon+O\left(\varepsilon^{2}\right) \\
v_{i}(\varepsilon)=v_{i}(0)+v_{i}^{\prime}(0) \varepsilon+O\left(\varepsilon^{2}\right)
\end{gathered}
$$

whose coefficients can be determined by substituting these expansions into $J(\varepsilon) v(\varepsilon)=\sigma(\varepsilon) v(\varepsilon)$ and equating coefficients of like powers of $\varepsilon$. This results in the (matrix algebraic) equations

$$
\begin{gathered}
\left(J(0)-\sigma_{i}(0) I\right) v_{i}(0)=0 \\
\left(J(0)-\sigma_{i}(0) I\right) v_{i}^{\prime}(0)=\sigma_{i}^{\prime}(0) v_{i}(0)-J^{\prime}(0) v_{i}(0) .
\end{gathered}
$$

By Eq. (6a) we see that $\sigma_{i}(0)$ is an eigenvalue of $J(0)$ and $v_{i}(0)$ is an associated eigenvector. The eigenvalues of $\mathrm{J}(0)$ turn out to be the roots of unity:

$$
\begin{aligned}
& \sigma_{1}(0)=1, \quad v_{1}(0)=\left(\begin{array}{c}
1 \\
1-\mu_{l} \\
1-\mu_{l}
\end{array}\right) \\
& \sigma_{2}(0)=\frac{1}{2}(-1+i \sqrt{3}), \quad v_{2}(0)=\left(\begin{array}{c}
-1-i \sqrt{3} \\
\left(1-\mu_{l}\right)(-1+i \sqrt{3}) \\
2\left(1-\mu_{l}\right)
\end{array}\right) \\
& \sigma_{3}(0)=\frac{1}{2}(-1-i \sqrt{3}), \quad v_{3}(0)=\left(\begin{array}{c}
-1+i \sqrt{3} \\
\left(1-\mu_{l}\right)(-1-i \sqrt{3}) \\
2\left(1-\mu_{l}\right)
\end{array}\right)
\end{aligned}
$$

Since to lowest order the eigenvalues have absolute value equal to 1 , the coefficients $\sigma_{i}^{\prime}(0)$ (if nonzero) determine the stability of the positive equilibrium for small $\varepsilon>0$.

Equation (6b) for $v_{i}^{\prime}(0)$ is solvable if and only if its right hand side is orthogonal to the eigenvector $w_{i}$ of the transpose

$$
\left(J(0)-\sigma_{i}(0) I\right)^{*}=\left(\begin{array}{ccc}
-\sigma_{i}(0) & 1-\mu_{l} & 0 \\
0 & -\sigma_{i}(0) & 1 \\
\frac{1}{1-\mu_{l}} & 0 & -\sigma_{i}(0)
\end{array}\right)
$$

This fact yields the formula [6].

$$
\sigma_{i}^{\prime}(0)=\frac{w_{i} \cdot J^{\prime}(0) v_{i}(0)}{w_{i} \cdot v_{i}(0)}
$$

(where "." denotes inner product). Our next step is to calculate $\sigma_{i}^{\prime}(0)$ for each of the three eigenvalues of $J(0)$. 
To calculate $\sigma_{1}^{\prime}(0)$ we first find

$$
w_{1}=\left(\begin{array}{c}
1-\mu_{l} \\
1 \\
1
\end{array}\right)
$$

and then use formula (7) to obtain a formula for $\sigma_{1}^{\prime}(0)$ that yields the expansion

$$
\sigma_{1}(\varepsilon)=1-\frac{1}{3}\left(c_{e l}+\left(c_{e a}+c_{p a}\right)\left(1-\mu_{l}\right)\right) \varepsilon+O\left(\varepsilon^{2}\right)
$$

for one of the eigenvalues of $J(\varepsilon)$, which we see is a real eigenvalue less than 1 in absolute value for $\varepsilon>1$ small.

To calculate $\sigma_{2}^{\prime}(0)$ we first find

$$
w_{2}=\left(\begin{array}{c}
2\left(1-\mu_{l}\right) \\
-1+i \sqrt{3} \\
-1-i \sqrt{3}
\end{array}\right)
$$

and use the formula (7) to obtain the formula

$$
\sigma_{2}^{\prime}(0)=-\frac{1}{3}\left(c_{e l}+\left(1-\mu_{l}\right) c_{p a}-\frac{1}{2} c_{e a}\left(1-\mu_{l}\right)+\frac{1}{2} c_{e a}\left(1-\mu_{l}\right) i \sqrt{3}\right) .
$$

The coefficient $\sigma_{3}^{\prime}(0)$ is the complex conjugate of $\sigma_{2}^{\prime}(0)$. These coefficients provide second order expansions for a complex conjugate pair of eigenvalues $\sigma_{2}(\varepsilon)$ and $\sigma_{3}(\varepsilon)$. A calculation shows the absolute value of this complex pair

$$
\left|\sigma_{2}\right|^{2}=\left|\sigma_{3}\right|^{2}=1+\frac{1}{3}\left(c_{e l}+c_{p a}\left(1-\mu_{l}\right)\right) \varepsilon+O\left(\varepsilon^{2}\right)
$$

is greater than 1 for $\varepsilon>0$ sufficiently small. The positive equilibrium is therefore unstable for $\varepsilon>0$ small (i.e. for $n-1>0$ near 0 ). In fact, we have shown the equilibrium is a saddle point possessing a two dimensional (local) unstable manifold and a one dimensional (local) stable manifold.

At first glance, the instability of the bifurcating positive equilibrium is perhaps unexpected, since transcritical bifurcations are generally associated with an exchange of stability. However, for the LPA model (2) the bifurcation at the trivial equilibrium is nongeneric in the sense that both a real and a complex conjugate pair of eigenvalues simultaneously leave the unit circle in the complex plane as $n$ increases through the bifurcation point $n=1(\varepsilon=0)$. The eigenvalue $\sigma_{1}(0)=1$ at this bifurcation is associated with the transcritical bifurcation of the equilibria. The complex pair $\sigma_{2}(\varepsilon), \sigma_{3}(\varepsilon)$, that leaves the unit circle at $\varepsilon=0$ suggests the bifurcation of an invariant loop [18,19]. However, the (Neimark-Sacker or discrete Hopf) theorem that supports this conclusion does not apply to the bifurcation occurring here. This is not only because of the simultaneous occurrence of the eigenvalue 1, but also because the conjugate pair leaves the unit circle at the cube roots of unity, which turns out to be one of the "resonance" cases omitted by the theorem [11,12]. Although this case has been studied [2,20], we do not need to refer to general theories since, as we see below, it is possible to analyze details of the bifurcation in a straightforward way. Not only will we show, under certain conditions, that an invariant loop bifurcates from 
the trivial equilibrium at $n=1$, but we will also obtain a detailed description its geometry and dynamic properties.

\section{SYNCHRONOUS ORBITS}

To study the existence of an invariant loop for Eq. (2) when $n>1$ we introduce the notion of synchronous orbits. We say a triple $\left(L_{t}, P_{t}, A_{t}\right)$ is synchronous at time $t$ if one of its components equals zero and at least one component is nonzero. It is not difficult to see from the Eq. (2) that an orbit synchronous at some time $t$ is in fact synchronous for all times $t \geq 0$. Thus, an orbit is synchronous (i.e. is synchronous for all $t$ ) if and only if its initial condition is synchronous. This means the nonnegative quadrants of the coordinate planes are invariant under the map defined by Eq. (2). In fact the motion in these nonnegative coordinate quadrants maps points from one coordinate quadrant to the next in sequential order. The same is true of the positive coordinate axes, the orbits in this case consisting of triples containing two zero components. Such orbits we call fully synchronous. An orbit is fully synchronous if and only if its initial condition is fully synchronous. This name comes from the biological interpretation of a fully synchronous orbit, namely, that the three life cycle stages are synchronized temporally in such a way that they never overlap.

Since the motion defined by Eq. (2) maps the nonnegative quadrants of the three coordinate planes into one another sequentially, we can study this motion using the second composite of the map. Let

$$
\left(\begin{array}{l}
x_{t} \\
y_{t} \\
z_{t}
\end{array}\right)
$$

denote the iterates of this composite (i.e. every third point of an orbit of Eq. (2)). From the Eq. (2) we find that the map

$$
\left(\begin{array}{l}
x_{t} \\
y_{t} \\
z_{t}
\end{array}\right) \rightarrow\left(\begin{array}{l}
x_{t+1} \\
y_{t+1} \\
z_{t+1}
\end{array}\right)
$$

is defined by the equations

$$
\begin{aligned}
& x_{t+1}=n x_{t} \exp [-c_{p a} y_{t} e^{-c_{p a} z_{t}}-c_{e a}\left(1-\mu_{l}\right) x_{t} \exp \left(-c_{p a} y_{t} e^{-c_{p a} z_{t}}\right) \\
&\left.-c_{e l} \frac{n}{1-\mu_{l}} y_{t} \exp \left(-c_{p a} z_{t}-c_{e a} y_{t} e^{-c_{p a} z_{t}}-c_{e l} \frac{n}{1-\mu_{l}} z_{t} e^{-c_{e a} z_{t}-c_{e l} x_{t}}\right)\right] \\
& y_{t+1}=n y_{t} \exp \left[-c_{p a} z_{t}-c_{e a} y_{t} e^{-c_{p a} z_{t}}-c_{e l} \frac{n}{1-\mu_{l}} z_{t} \exp \left(-c_{e a} z_{t}-c_{e l} x_{t}\right)\right] \\
& z_{t+1}=n z_{t} \exp \left[-c_{e a} z_{t}-c_{e l} x_{t}-c_{p a}\left(1-\mu_{l}\right) x_{t} \exp \left(-c_{p a} y_{t} e^{-c_{p a} z_{t}}\right)\right]
\end{aligned}
$$

From these equations we see that if any component initially equals 0 then it remains equal to 0 for all $t$. To study the dynamics of the composite map on the nonnegative 
quadrant of, say, the $x, z$-coordinate plane, we take $y_{0}=0$. These dynamics are described by the two dimensional system

$$
\begin{gathered}
x_{t+1}=n x_{t} \exp \left(-c x_{t}\right) \\
z_{t+1}=\left[n \exp \left(-\alpha x_{t}\right)\right] z_{t} \exp \left(-\beta z_{t}\right)
\end{gathered}
$$

of difference equations for the components $x_{t}$ and $z_{t}$, where the coefficients

$$
c=c_{e a}\left(1-\mu_{l}\right), \quad \alpha=c_{e l}+c_{p a}\left(1-\mu_{l}\right), \quad \beta=c_{e a}
$$

are positive.

A $p$-cycle is periodic orbit of (minimal) period $p$. Fixed points (1-cycles) of the two dimensional maps (9a) and (9b) correspond to synchronous 3-cycles of the LPA model (2). Notice the first equation (9a) is uncoupled from the second equation (9b). The first equation is in fact the famous Ricker map, about whose dynamics a great deal is known. The $x$-component of every orbit of Eqs. (9a) and (9b) satisfies the Ricker map (9a) and, given a solution of this map, the second equation (9b) becomes a nonautonomous version of the Ricker map. If $n<1$, then $\lim x_{t}=0$. As a result, Eq. (9b) is asymptotically autonomous and its limiting equation is the Ricker equation $v_{t+1}=n v_{t} \exp \left(-\beta v_{t}\right)$, all of whose solutions satisfy $\lim v_{t}=0$. By Theorem A.1 in Appendix A, it follows that $\lim z_{t}=0$. This result is consistent with Theorem 1 .

Fully synchronous orbits of the LPA model (2) correspond to initial conditions $z_{0}=0$ for the two dimensional systems (9a) and (9b). Thus, the dynamics of fully synchronous orbits of Eq. (2) are governed by the Ricker equation (9a). For $n>1$ the Ricker map has a (unique) positive equilibrium $x=c^{-1} \ln n$. Therefore, for $n>1$ there exists a fully synchronous 3-cycle of Eq. (2). As $n$ increases the dynamics of the Ricker map undergo the familiar period doubling route to chaos. A 2-cycle for the Ricker map corresponds to a fully synchronous 6-cycle of Eq. (2), a 4-cycle corresponds to a fully synchronous 12-cycle of Eq. (2), and so on.

The attractors of the Ricker map correspond to attractors of the LPA model (2) when initial conditions are restricted to the coordinate axes. For example, if

$$
1<n<e^{2}
$$

then

$$
\left(\begin{array}{l}
x \\
z
\end{array}\right)=\left(\begin{array}{c}
\frac{1}{c} \ln n \\
0
\end{array}\right)
$$

is a LAS equilibrium of Eqs. (9a) and (9b) and globally attracts all positive initial conditions in the $x$, $z$-plane. This equilibrium corresponds to the fully synchronous 3 -cycle of the LPA equation (2) consisting of the vectors

$$
\left(\begin{array}{c}
\frac{1}{c_{e a}\left(1-\mu_{l}\right)} \ln n \\
0 \\
0
\end{array}\right) \rightarrow\left(\begin{array}{c}
0 \\
\frac{1}{c_{e a}} \ln n \\
0
\end{array}\right) \rightarrow\left(\begin{array}{c}
0 \\
0 \\
\frac{1}{c_{e a}} \ln n
\end{array}\right)
$$

This 3-cycle is a global attractor on the coordinate axes, i.e. it is LAS with respect to fully synchronous orbits and globally attracts all positive, fully synchronous orbits. We can make a similar translation from other kinds of Ricker attractors for other ranges of $n$ 
(including chaotic ranges) to attractors of the LPA model (2) restricted to fully synchronous orbits. We summarize these observations in the following theorem.

THEOREM 2 For $1<n<e^{2}$, the LPA model (2) has a unique, nontrivial fully synchronous 3-cycle given by Eq. (11). This 3-cycle attracts all fully synchronous orbits of Eq. (2). For $n>e^{2}$ the system has a period doubling cascade of fully synchronous $\left(3 \times 2^{n}\right)$-cycle attractors and, for sufficiently large n, has "fully synchronous chaotic" attractors. Here by "attractor" we mean with respect only to fully synchronous orbits.

Incidentally, this result provides the first proof of chaotic dynamics for the LPA model (1). However, the constraint $\mu_{a}=1$ does not permit an application to the chaos experiment and study described in Refs. $[4,8,10]$ where $\mu_{a}=0.96$. Indeed, in that application the chaotic attractor is not synchronous.

Next we turn our attention to synchronous orbits of the LPA model (2) that are not necessarily fully synchronous (i.e. to motion in the coordinate planes). This we do by considering the composite map (8) restricted to the coordinate planes. Without loss in generality, we give details only for the $x$, $z$-plane on which the dynamics are given by the Eqs. (9a) and (9b). This system has four equilibria

$$
\left(\begin{array}{l}
x \\
z
\end{array}\right)=\left(\begin{array}{l}
0 \\
0
\end{array}\right), \quad\left(\begin{array}{c}
\frac{1}{c} \ln n \\
0
\end{array}\right), \quad\left(\begin{array}{c}
0 \\
\frac{1}{\beta} \ln n
\end{array}\right)
$$

and

$$
\left(\begin{array}{c}
\frac{1}{c} \ln n \\
\frac{\beta\left(1-\mu_{l}\right)-\alpha}{c \beta} \ln n
\end{array}\right)
$$

The three equilibria (12) are nonnegative for $n>1$. The first equilibrium corresponds to the trivial equilibrium of the LPA model and the second equilibrium corresponds to the fully synchronous 3-cycle (11). The third equilibrium also yields a fully synchronous 3 -cycle, but it is just a phase shift of the cycle (11). Finally, if $\beta\left(1-\mu_{l}\right)-\alpha>0$ then the equilibrium (13) corresponds to a synchronous, nonnegative 3-cycle of Eq. (11) that is not fully synchronous (i.e. a partially synchronous 3-cycle in which only one component equals zero at any given $t$ ). We will not study this case here and instead assume

$$
\beta\left(1-\mu_{l}\right)-\alpha<0
$$

or, in terms of the original LPA model coefficients,

$$
\left(c_{p a}-c_{e a}\right)\left(1-\mu_{l}\right)+c_{e l}>0 .
$$

Under this assumption only the equilibria (12) are nonnegative and the only synchronous 3-cycle of the LPA model (2) (up to phase shifts) is Eq. (11).

This assumption on the coefficients in the LPA model is not biologically unreasonable; it is, for example, satisfied by the estimated coefficients used in the chaos experiment based on the LPA model $[8,10]$.

Lemma 3 Assume (10) and (14) hold. Then the equilibrium

$$
\left(\begin{array}{l}
x \\
z
\end{array}\right)=\left(\begin{array}{c}
\frac{1}{c} \ln n \\
0
\end{array}\right)
$$


of (9a) and (9b) is LAS in the $x, z$-plane and attracts all orbits in the positive $x, z$ quadrant.

Proof From Eq. (9a) it follows that $x_{0}>0$ implies $\lim x_{t}=c^{-1} \ln n$. Equation (9b) is an asymptotically autonomous difference equation whose limiting equation is another Ricker equation, namely,

$$
v_{t+1}=\left[n \exp \left(-\alpha c^{-1} \ln n\right)\right] v_{t} \exp \left(-\beta v_{t}\right) .
$$

Algebraic calculations show, under the stated assumptions, that

$$
n \exp \left(-\alpha c^{-1} \ln n\right)<1
$$

and hence it follows that $\lim v_{t}=0$ for all $v_{0} \geq 0$. Theorem A.1 in Appendix A implies $\lim z_{t}=0$ for all $z_{0} \geq 0$.

The local asymptotic stability follows from a calculation of the Jacobian of Eqs. (9a) and (9b) and its evaluation at the equilibrium. The eigenvalues turn out to be

$$
1-\ln n, \quad n^{-\left[\left(c_{p a}-c_{e a}\right)\left(1-\mu_{l}\right)+c_{e l}\right] / c_{e a}\left(1-\mu_{l}\right)} .
$$

Both are less than one in absolute value under the stated assumptions.

Similar arguments using the composite equation (8) restricted to the other two coordinate planes yield the similar global stability results in those planes.

Lemma 4 Assume (10) and (14) hold. Then the equilibrium

$$
\left(\begin{array}{l}
x \\
y
\end{array}\right)=\left(\begin{array}{c}
0 \\
\frac{1}{\beta} \ln n
\end{array}\right)
$$

is LAS in the $x, y$-plane and attracts all orbits in the positive $x, y$-quadrant. Also, the equilibrium

$$
\left(\begin{array}{l}
y \\
z
\end{array}\right)=\left(\begin{array}{c}
0 \\
\frac{1}{\beta} \ln n
\end{array}\right)
$$

is LAS in the $y$, z-plane and attracts all orbits in the positive y, z-quadrant.

The three equilibria in Lemmas 3 and 4 provide three equilibria

$$
\left(\begin{array}{c}
x \\
y \\
z
\end{array}\right)=\left(\begin{array}{c}
\frac{1}{c} \ln n \\
0 \\
0
\end{array}\right),\left(\begin{array}{c}
0 \\
\frac{1}{\beta} \ln n \\
0
\end{array}\right),\left(\begin{array}{c}
0 \\
0 \\
\frac{1}{\beta} \ln n
\end{array}\right)
$$

of the composite equation (8). These equilibria correspond to the three different phases of the fully synchronous 3-cycle (11) of the LPA model (2). Lemmas 3 and 4 imply the following restricted global stability result for this 3-cycle.

THEOREM 5 Assume $1<n<e^{2}$ and $\left(c_{p a}-c_{e a}\right)\left(1-\mu_{l}\right)+c_{e l}>0$. Then the fully synchronous 3-cycle (11) attracts all synchronous orbits of the LPA model (2).

The next result shows, however, that the 3 -cycle is unstable as an orbit of the LPA model (2). 
TheOREM 6 Assume $1<n<e^{2}$ and $\left(c_{p a}-c_{e a}\right)\left(1-\mu_{l}\right)+c_{e l}>0$. Then the fully synchronous 3-cycle (11) is unstable (it is in fact a saddle).

Proof We can determine the local stability properties of the 3-cycle by finding the eigenvalues of the Jacobian J of composite map (8) evaluated at any one of the equilibria (15). By the chain rule this Jacobian is the product $J=J_{3} J_{2} J_{1}$ where the matrices $J_{1}, J_{2}, J_{3}$ are obtained by evaluating the Jacobian of the LPA model (2), namely, the matrix

$$
\left(\begin{array}{ccc}
-c_{e l} \frac{n}{1-\mu_{l}} A e^{-c_{e a} A-c_{e l} L} & 0 & \left(1-c_{e a} A\right) \frac{n}{1-\mu_{l}} e^{-c_{e a} A-c_{e l} L} \\
1-\mu_{l} & 0 & 0 \\
0 & e^{-c_{p a} A} & -c_{p a} P e^{-c_{p a} A}
\end{array}\right)
$$

at each of the points of the 3-cycle (11) [1]. This straightforward calculation yields the triangular matrix

$$
J=\left(\begin{array}{ccc}
1-\ln n & \frac{(\ln n-1)\left(1-\mu_{l}\right) c_{p a}-c_{e l} n}{c_{e a}\left(1-\mu_{l}\right)^{2}} \ln n & 0 \\
0 & n & 0 \\
0 & 0 & n^{-\left(\left(c_{p a}-c_{e a}\right)\left(1-\mu_{l}\right)+c_{e l}\right) / c_{e a}\left(1-\mu_{l}\right)}
\end{array}\right)
$$

whose eigenvalues appear along the diagonal. Since the second eigenvalue $n>1$, the 3-cycle is unstable. The assumption $1<n<e^{2}$ implies the first eigenvalue satisfies $|1-\ln n|<1$. The inequality (14) implies the third (real and positive) eigenvalue is less than one. Thus, the 3-cycle has a two dimensional stable manifold (on which the motion is locally a stable node).

The complex eigenvalues associated with the positive equilibrium (see the proof of Theorem 1) imply that the orbits of the LPA model (2), at least near the equilibrium, have an outward spiral motion. In the next section, we see how an invariant loop in the coordinate planes, on which the fully synchronous 3-cycle resides, can be the attractor of these orbits.

\section{CYCLE CHAINS}

Under the conditions of Theorem 5 the 3-cycle (11) globally attracts all nontrivial, nonnegative synchronous orbits of Eq. (2). We now describe the dynamics of synchronous cycles in more detail. In the nonnegative quadrant of the $x$, $z$-plane the Eqs. (9a) and (9b) have three equilibria. Under the conditions of Theorem 5 the equilibrium

$$
\left(\begin{array}{l}
x \\
z
\end{array}\right)=\left(\begin{array}{c}
\frac{1}{c} \ln n \\
0
\end{array}\right)
$$

is globally attracting in the quadrant (Lemma 3). The trivial equilibrium

$$
\left(\begin{array}{l}
x \\
z
\end{array}\right)=\left(\begin{array}{l}
0 \\
0
\end{array}\right)
$$


is a repellor, as can be seen by a straightforward calculation of the eigenvalues of the Jacobian of Eqs. (9a) and (9b) evaluated at this equilibrium (both equal to $n>1$ ). The third nonnegative equilibrium

$$
\left(\begin{array}{c}
0 \\
\frac{1}{\beta} \ln n
\end{array}\right)
$$

is a saddle. This is because the eigenvalues of the Jacobian at this point are $n>1$ and $1-\ln n$, whose absolute value is less than one by assumption. If $x_{0}=0$ in Eqs. (9a) and (9b), then $x_{t}=0$ for all $t$ and the resulting equation $z_{t+1}=n z_{t} \exp \left(-\beta z_{t}\right)$ for $z_{t}$ implies $\lim z_{t}=$ $\beta^{-1} \ln n$. Thus, the $z$-axis is the stable manifold of the saddle equilibrium (17). This saddle also has a (locally, one dimensional) unstable manifold [6]. This manifold is (forward) invariant and contains backward orbits that tend to the equilibrium point. The branch lying in the positive quadrant contains orbits that, by Lemma 3, tend to the globally attracting equilibrium (16). Thus, we have a "heteroclinic" invariant set that connects (i.e. contains orbits that connect) the two equilibria (16) and (17). A similar analysis holds in the positive quadrants of the other two coordinate planes.

In three dimensions, the three equilibria (15) and their planar hetero-clinic connectors described above form an invariant loop for the composite map (8) that lies in the coordinate planes (see Fig. 1a).

Recall that with regard to the LPA model (2) the three equilibria (15) of the composite map correspond to the three phases of the fully synchronous 3-cycle (11). The cycle chain of the composite map in Fig. 1a yields the following theorem about the LPA model.

THEOREM 7 Assume $1<n<e^{2}$ and $\left(c_{p a}-c_{e a}\right)\left(1-\mu_{l}\right)+c_{e l}>0$. There exists an invariant loop of the LPA model (2) consisting of synchronous orbits (i.e. the loop lies in the nonnegative quadrants of the coordinate planes). The loop has the form of a "cycle chain" in
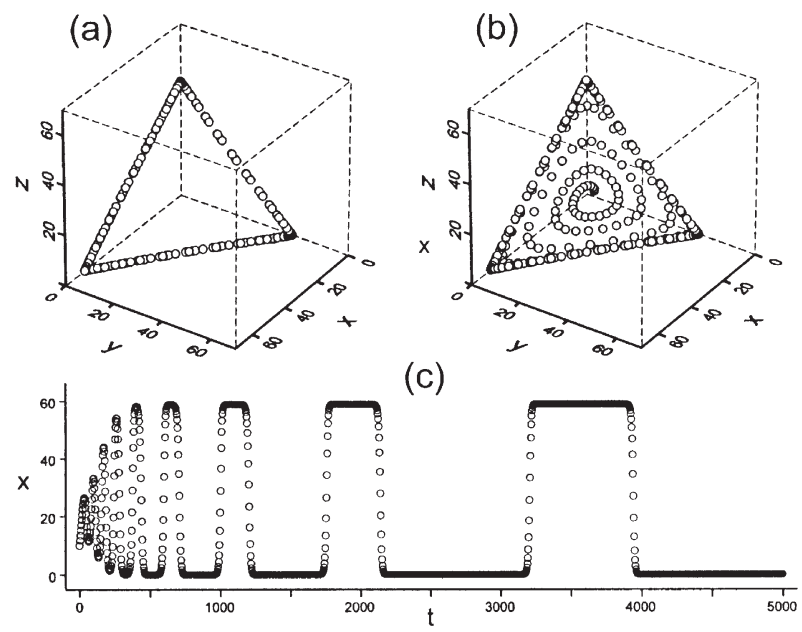

FIGURE 1 (a) The synchronous cycle chain of the composite system (8) is shown for $c_{e l}=c_{e a}=c_{p a}=0.1$ $\mu_{l}=0.2$ and $b=2(n=1.6)$. (b) An orbit of Eq. (8) approaches the cycle chain in (a) in an outwardly spiralling manner. The initial conditions are $x_{0}=y_{0}=z_{0}=10$. (c) The $x$ component of the orbit in (b). 
that it is made up of the 3-cycle (11) and invariant sets of synchronous orbits that heteroclinically connect the three phases of the 3-cycle.

We saw in Theorem 6 that the 3-cycle lying on the cycle chain is unstable as an orbit of the LPA model. However, the cycle chain described in Theorem 7 can be locally attracting and we conjecture this is the case, at least for $n-1>0$ sufficiently small. (The cycle chain cannot be globally attracting because the LPA model has a positive equilibrium with a stable manifold). Figure 2 shows an example that supports this conjecture.

Orbits of the composite system (8) that approach the composite cycle chain exhibit episodes of near equilibrium dynamics as they sequentially visit the vicinity of the three saddle equilibria. Figures $1 \mathrm{~b}$ and $\mathrm{c}$ show an example. The duration of these episodes increases without bound as time increases, since each visit is closer than the previous and hence each "flyby" of the saddle lasts longer than the previous one. (This is reminiscent of a famous example in theoretical ecology due to May and Leonard [16], although the context in that example-Lotka/Volterra dynamics for three competing species-is completely different.) The corresponding orbits of the LPA model (2) show episodes of near 3-cycle dynamics that sequentially resemble the three phase shifts of the 3-cycle (11). The duration of these episodes increases without bound as time increases. Thus, the orbit asymptotically resembles the 3-cycle, although there are repeated interruptions-of increasing temporal separation-that result in phase shifts in the oscillations (see Fig. 3).
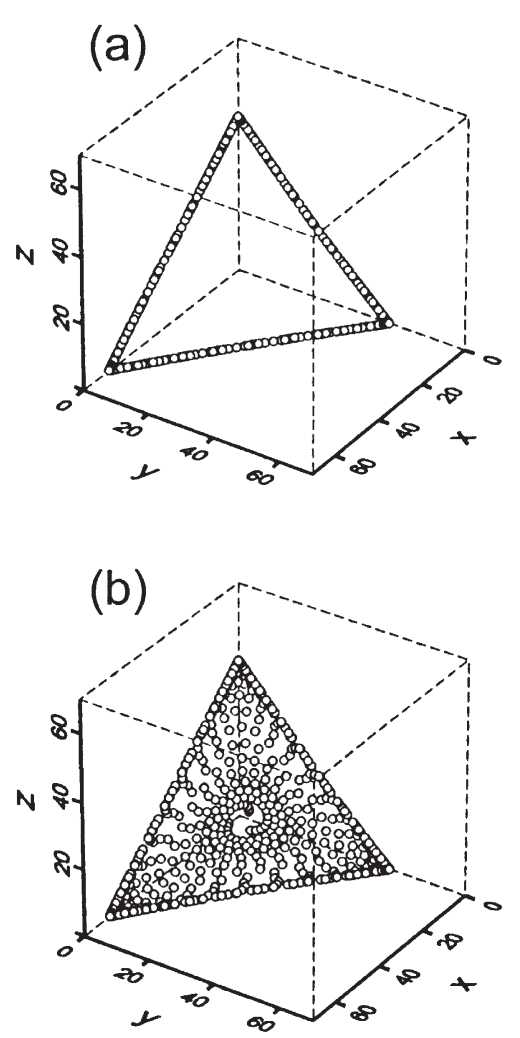

FIGURE 2 (a) Shown is the synchronous cycle chain for the LPA model (2) that corresponds to the cycle chain for the composite system (8) in Fig. 1a. (b) The orbit of Eq. (2) with initial conditions $L_{0}=P_{0}=A_{0}=10$ approaches the cycle chain in (a) in an outwardly spiraling manner. 


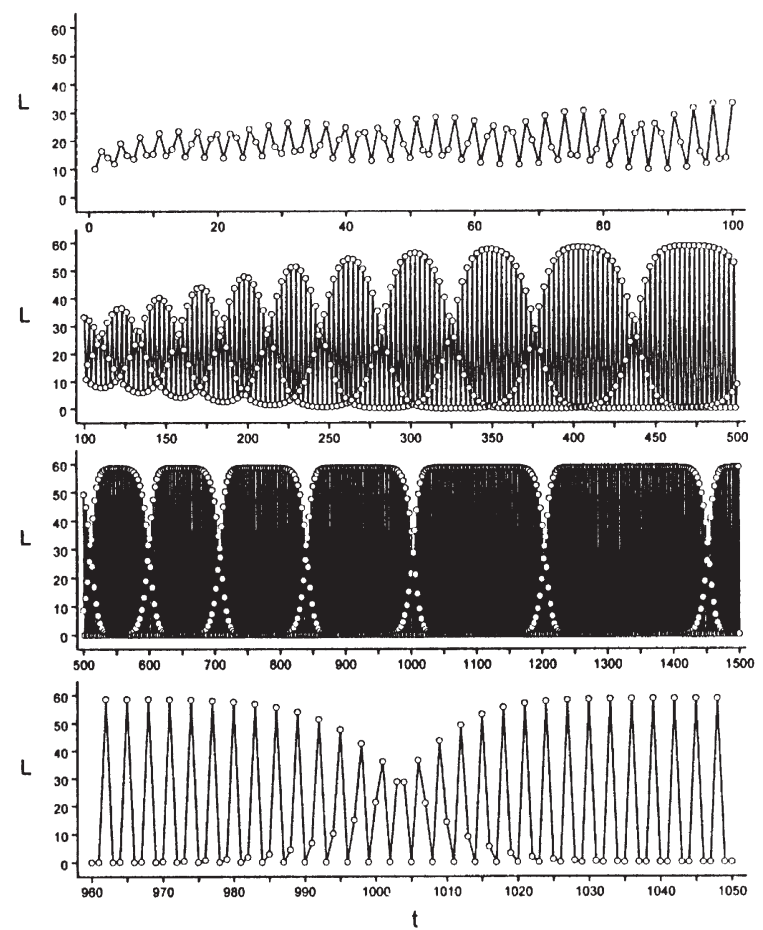

FIGURE 3 Shown are time series plots of the $L$ component of the orbit in Fig. $2 b$ that approaches the cycle chain in Fig. 2a. The top two plots show transients as the orbit spirals slowly outward towards the cycle chain. The third plot shows the "3-cycle-like" episodes of increasing duration caused by the sequential visits to the three phases of the 3 -cycle (11). The bottom plot zooms into view one of the "chicken" or "stutter" steps that occurs as the orbits leaves one (saddle) phase of the 3 -cycle and moves to the next phase.

It is interesting to note that oscillatory phase shifts have been observed in flour beetle dynamics and these shifts have been analyzed using the LPA model [14]. In these previous studies the mechanism causing the phase shifts is stochastic, however, and not deterministic, as it is here.

\section{CONCLUDING REMARKS}

We have seen that some complicated dynamics of the special case (2) of the LPA model (1) are tractable to analysis. Many questions remain, however. There is the problem of determining under what conditions the synchronous cycle chain in Theorem 7 is an attractor in three dimensions. Another open problem is the case $\left(c_{p a}-c_{e a}\right)\left(1-\mu_{l}\right)+c_{e l}<0$ when the 3 -cycle is only partially synchronous. Also left open is a rigorous treatment of higher order cycle chains as $n$ is increased above $e^{2}$ and the Ricker equation undergoes a period doubling cascade. A natural conjecture is that higher period cycles of the Ricker map produce more complicated cycle chains which consist of heteroclinic connections of all phase shifts of the cycle. An example appears in Fig. 4, where a value of $n$ is chosen that produces a 2-cycle of the Ricker map and hence a 6-cycle of the LPA model. In this figure one can see the 6-cycle and the invariant connective sets. Also of interest would be a treatment of synchronous chaotic attractors resulting from sufficiently large values of $n$. 

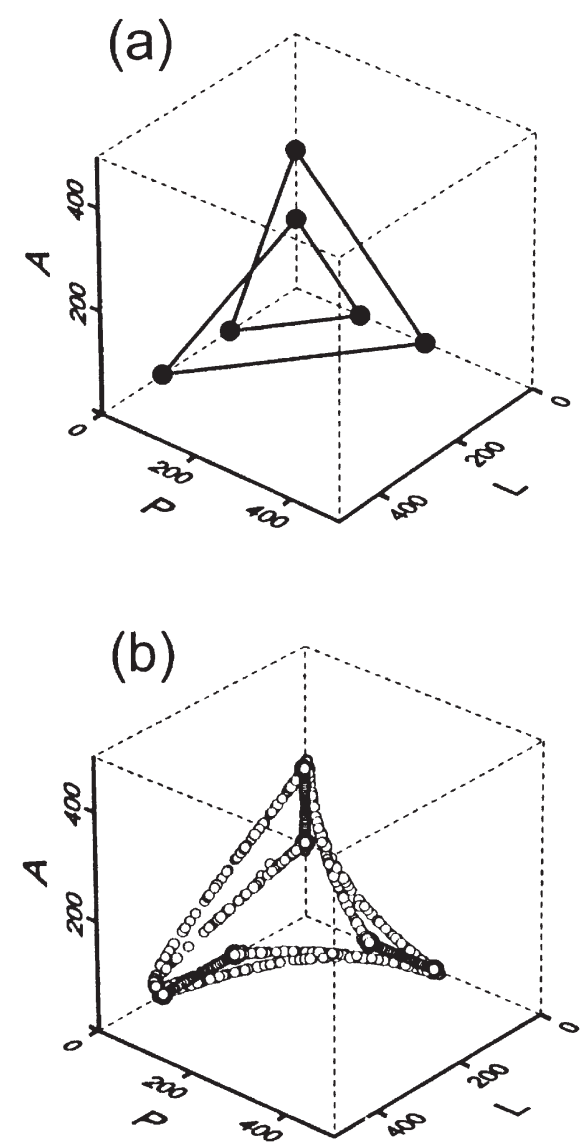

FIGURE 4 (a) If in Fig. 1 the parameter $b$ is increased to 10 (hence $n$ is increased to 8), the equilibrium of the Ricker equation (9a) destabilizes and orbits approach a 2-cycle. This fully synchronous 2-cycle of the composite system (8) corresponds to the fully synchronous 6-cycle of the LPA model (2) shown here. (b) The phases of the 6-cycle in (a), together with the connecting heteroclinic (synchronous) invariant sets, form an attractor for Eq. (2). This plot of the attractor was found numerically by calculating several orbits that converge to the attractor and discarding their initial transients.

\section{Acknowledgements}

This research was supported by National Science Foundation grant DMS 9973126.

\section{References}

[1] K. T. Alligood, T. D. Sauer and J. A. Yorke, Chaos: An Introduction to Dynamical Systems, Springer, Berlin, 1997.

[2] V. I. Arnold, Loss of stability of self oscillations close to resonances and versal deformations of equivariant vector fields, Funct. Anal. Appl., 11 (1977), 1-10.

[3] H. Caswell, Matrix Population Models: Construction, Analysis and Interpretation, Second edition, Sinauer Associates, Inc. Publishers, Sunderland, MA, 2001.

[4] R. F. Costantino, R. A. Desharnais, J. M. Cushing and B. Dennis, Chaotic dynamics in an insect population, Science, 275 (1997), 389-391.

[5] J. M. Cushing, A strong ergodic theorem for some nonlinear matrix models for the dynamics of structured populations, Nat. Res. Modeling, 3 (1989), 331-357.

[6] J. M. Cushing, An Introduction to Structured Population Dynamics, CBMS-NSF Regional Conference Series in Applied Mathematics, SIAM, Philadelphia, Vol. 71, (1998).

[7] J. M. Cushing, R. F. Costantino, B. Dennis, R. A. Desharnais and S. M. Henson, Nonlinear population dynamics: models, experiments and data, J. Theor. Biol., 194(1) (1998), 1-9. 
[8] J. M. Cushing, R. F. Costantino, B. Dennis, R. A. Desharnais and S. M. Henson, Chaos in Ecology: Experimental Nonlinear Dynamics, Academic Press (an imprint of Elsevier), New York, 2003.

[9] B. Dennis, R. A. Desharnais, J. M. Cushing and R. F. Costantino, Non-linear demographic dynamics: mathematical, models, statistical methods, and biological experiments, Ecol. Monogr., 65(3) (1995), 261-281.

[10] B. Dennis, R. A. Desharnais, J. M. Cushing, M. Shandelle and R. F. Costantino, Estimating chaos and complex dynamics in an insect population, Ecol. Mongr., 71(2), (2001), 277-303.

[11] S. N. Elaydi, Discrete Chaos, Chapman \& Hall/CRC press, New York, 1999.

[12] J. Guckenheimer and P. Holmes, Nonlinear Oscillations, Dynamical Systems and Bifurcations of Vector Fields, Springer, Berlin, 1983.

[13] J. W. Haefner, Modeling Biological Systems: Principles and Applications, Chapman \& Hall, International Thomson Publishing, New York, 1996.

[14] S. M. Henson, J. M. Cushing, R. F. Costantino, B. Dennis and R. A. Desharnais, Phase switching in biological population, Proc. R. Soc., 265 (1998), 2229-2234.

[15] Y. Kuang and J. M. Cushing, Global stability in a nonlinear difference-delay equation model of flour beetle population growth, J. Diff. Eqs. Appl., 2 (1996), 31-37.

[16] R. M. May and W. J. Leonard, Nonlinear aspects of competition between three species, SIAM J. Appl. Math., 29 (1975), 243-253.

[17] L. D. Mueller and A. Joshi, Stability in Model Populations Monographs in Population Biology 31, Princeton University Press, Princeton, NJ, 2000.

[18] Y. Neimark, On some cases of periodic motions depending on parameters, Dokl. Acad. Nauk. SSSR, 129 (1959), 736-739.

[19] R. S. Sacker, A new approach to the perturbation theory of invariant surfaces, Comm. Pure Appl. Math., 18 (1965), 717-732.

[20] F. Takens, Forced oscillations and bifurcations, Comm. Math. Inst. Rijkuniversiteit Utrecht, 3 (1974), 1-59.

\section{APPENDIX A}

For convenient reference, we state below a known theorem, concerning asymptotically autonomous maps, that we use in the proofs of Theorem 2 and Lemma 3. Consider the nonautonomous, scalar difference equation

$$
u_{t+1}=f\left(t, u_{t}\right)
$$

where $f(t, \cdot): R \rightarrow R$ is continuously differentiable for each $t=0,1,2 \ldots$.

Assume

H1 : $\quad f(t, \cdot) \rightarrow g(\cdot)$ uniformly on compact subsets of $R$ as $t \rightarrow \infty$ and $g: R \rightarrow R$ is continuously differentiable.

The autonomous scalar difference equation

$$
v_{t+1}=g\left(v_{t}\right)
$$

is called the limiting equation associated with Eq. (A.1). Assume

$\mathrm{H} 2$ : $\quad$ in any compact subset of $R$ the limiting equation (A.2) has at most a finite number of cycles, all of which are hyperbolic, and every bounded solution of equation (A.2) is either a cycle or tends asymptotically to a cycle.

THeOREM A.1 [5] Under assumptions H1 and H2, every bounded solution of Eq. (A.1) asymptotically approaches a cycle of the limiting equation (A.2).

It is not necessarily true that the cycle approached by an orbit of Eq. (A.1) is an attracting or LAS cycle of the limiting equation (A.2). See Ref. [5] for examples and for other results concerning the asymptotic dynamics of asymptotically autonomous maps. 